\title{
Personal Digital Health Assistants
}

Uday Jain

Research in anesthesiology, Professor of anesthesiology, San Mateo Medical Center, San Mateo, USA.

Corresponding Author: Uday Jain, Research in anesthesiology, Professor of anesthesiology, San Mateo Medical Center, San Mateo, USA.

\section{Received Date: 29 August 2021 | Accepted Date: 15 December 2021 | Published Date: 03 January 2022}

Citation: U Jain. (2022). Personal Digital Health Assistants. J. Clinical Surgery and Research. 3(1); DOI: 10.31579/2768-2757/027

Copyright: (02022 Uday Jain, This is an open-access article distributed under the terms of the Creative Commons Attribution License, which permits unrestricted use, distribution, and reproduction in any medium, provided the original author and source are credited.

\begin{abstract}
Personal Digital Health Assistants (PDHA) are applications that can run on virtually any computer or mobile device including a smart phone/ personal digital assistant (PDA). A PDHA acquires, stores, and analyzes health related information of an individual. It usually communicates with remote servers of a large organization which can connect it to various resources. PDHAs are an integral part of telehealth. Their utility has increased manyfold since the start of the pandemic. The systems are increasingly more complex and are involved in all aspects of care. The PDHA are usually utilized by patients or their caregivers with assistance from professionals. Many automated systems can be utilized free of charge. Some are available as part of a prepaid health plan.
\end{abstract}

Keywords: personal digital health assistants; personal digital assistant

\section{Introduction:}

Personal Digital Health Assistants (PDHA) are applications that can run on virtually any computer or mobile device including a smart phone/ personal digital assistant (PDA). A PDHA acquires, stores, and analyzes health related information of an individual. It usually communicates with remote servers of a large organization which can connect it to various resources. PDHAs are an integral part of telehealth. Their utility has increased manyfold since the start of the pandemic. The systems are increasingly more complex and are involved in all aspects of care. The PDHA are usually utilized by patients or their caregivers with assistance from professionals. Many automated systems can be utilized free of charge. Some are available as part of a prepaid health plan.

Increasing number of patients that anesthesiologists care for, utilize a PDHA. Additionally, anesthesiologists may utilize PDHAs for diagnosing and managing patients as discussed below.

\section{Artificial Intelligence}

Artificial intelligence (AI) has substantially increased the power and utility of PDHAs and telehealth. Large medical databases have been accumulated by some medical insurers, national health services, and medical institutions. These have been used to train AI systems to mimic diagnoses and management decisions of physicians.

These systems utilize neural networks and machine learning. Some utilize branching logic or statistical inference. AI based systems are superior to older keyword or rules-based systems in which each symptom is associated with a particular disease with an assigned probability. Training of these systems is usually ongoing and requires input from medical and information technology personnel.

\section{User Interface}

The patient can consult with the clinician via text, voice, or video on a HIPAA compliant connection. Widely utilized tele-conferencing services like Zoom have HIPAA compliant versions. Symptoms and other information may be entered by selecting from a list. Many systems also communicate by natural language text messages. Some allow natural language voice input and integration with digital voice assistants such as Amazon Alexa, Microsoft Cortana, and Google Assistant. Natural language input may be parsed into a list of statements the system understands. The user can choose the desired statements. Integration with services such as Google Cloud allows for real-time captioning and language translation. Some systems are available in multiple languages.

Voice and video consults can be live, i.e., real time, or asynchronous, i.e., recorded. Instead of projecting the physician's image on the patient's screen, some systems have the option of projecting a file photograph, an avatar, or another image. Some patients are more willing to reveal their mental and other health information in that setting.

Voice recognition may allow these systems to listen in on the physician patient consult and suggest differential diagnosis and management. This can be done in real time or off-line. Some systems allow digital input of data acquired by various physiological sensors and monitoring systems.

\section{Symptom Checkers}

One of PDHA's tasks is to develop a differential diagnosis based on symptoms and other information. In this role it has been referred to as a symptom checker. In the perioperative period, anesthesiologists usually care for patients with established diagnoses. Many patients are familiar with their chronic conditions such as hypertension, diabetes, and 
cardiovascular disease and are receiving care. However, in the settings of preoperative clinic, perioperative surgical home, pain clinic, and intensive care unit, anesthesiologists may be called upon to diagnose and manage their patients.

Like the public, anesthesiologists can perform Google and other web search of the relevant information. Every day, over one billion of Google searches are health-related ${ }^{1}$. However, such a search may not integrate all the information that is known or can be elicited, and is usually not adequate for clinical management. PDHAs provide diagnosis decision support that is superior to internet searches.

After the symptoms and other information are entered, the system asks a series of questions. Each question depends on the answer to the previous question. Usually only a few questions are asked. They consider the patient's demographics and medical history. Some may also consider vital signs, input from physiological sensors, and laboratory values. The systems provide the differential diagnoses and management plans. They assist the physician in considering the full range of differential diagnoses and avoid missing a potential diagnosis.

These systems have become practical tools for clinical diagnosis. They are most useful in pre-primary care setting. They pre-diagnose, triage, and can connect the patient to the appropriate medical facilities, providers, support services, and resources. They can assist call centers and emergency departments in triaging. They are most useful in diagnosing conditions that are not common.

\section{Clinician Consult}

Automated systems serve only in an advisory role. No doctor-patient relationship is established. They cannot make management decisions or order prescriptions. Many systems provide the option to pass information to in-house or outside physicians who manage the patient, usually for a fee or as part of a prepaid plan.

\section{Acute Care}

In response to the pandemic, US Centers for Medicare and Medicaid Services initiated "Hospitals without Walls" allowing care outside hospitals, in locations such as designated hotels. In November 2020, it expanded to "Acute Hospital Care at Home." These regulatory changes along with advances in technology have greatly increased the utilization of telehealth. Organizations such as Mayo Clinic and Kaiser Permanente are utilizing it to provide at home acute care previously provided in inpatient settings. Frequent detailed interaction between the patient and the health system, facilitated by the PDHA is critical for this purpose.

\section{Chronic Care}

Most of the systems participate in chronic care. They can prompt the user to collect clinical data on an ongoing basis. Data can be acquired from sensors and monitors. Data can be analyzed, transmitted, and shared with all the stakeholders. The systems can prompt the patients to follow therapeutic recommendations such as taking their medications. The systems can record the responses. They have become important tools in providing longitudinal care.

\section{Mental Health}

During the pandemic, much of the clinician provided mental health care is via PDHAs. This is likely to continue in the future. Many fully automated systems are also available to interact with clients.

\section{Well Care and Education}

PDHAs are playing an important role in preventive care and health maintenance. Smart phone applications that track physical activity are utilized widely. PDHAs can provide individualized instructions on life- style choices. PDHAs are also suitable for providing information about alternative and integrative medicine. Many systems have their own libraries and can also be used to access outside libraries.

\section{Education for Clinicians}

Some systems have a clinical educator mode which combines a differential diagnosis generator with case histories. It is useful for teaching clinical reasoning and medical diagnosis to clinicians. Links to medical literature relevant to the diagnoses are provided.

\section{Interaction with Electronic Medical Records}

Information in the electronic medical records (EMR) is usually entered by clinicians and facilities such as laboratories and is deemed reliable. Patients can usually access some of the information in the EMR without being able to alter it. Many entries in PDHAs are made by patients and caregivers. PDHAs may contain more information than EMRs, especially during the period the patient is in the usual state of health. Clinicians can extract reliable and relevant information from the PDHAs and include it in the EMR. Some PDHAs provide an application programming interface to facilitate communication with EMRs and other systems.

\section{Limitations}

Usually, these systems are proprietary. Interoperability and communication with other PDHAs and EMRs are limited. They are generally not regulated by public bodies. They have not been adequately validated by independent studies. Accuracy figures are usually not available [3]. Because they are frequently updated, it is difficult to evaluate them with studies that can take months. The results provided by any of these systems can be incorrect and should be verified by a clinician [4]. When used for triaging, these systems tend to be risk averse and are likely to triage the patient to get unnecessary further care [3]. When used for determining differential diagnoses, their utility is limited if they provide a long list of possible conditions.

\section{Available Systems}

Hundreds of systems are available. A few of the prominent ones are listed below.

Amwell.com. It connects 72 thousand providers to 80 million members. It assists in all stages of care.

teladochealth.com. It is used in the US and worldwide. It provides comprehensive services to 52 million members. It includes Livongo which focuses on chronic disease management.

ada.com. This German system is used in many countries by over 11 million users. It uses text chat on a mobile device.

buoyhealth.com. Developed through the Harvard Innovation Laboratory, the company's algorithm was initially trained on data from 18 thousand medical papers, 5 million patients, and 1,700 health conditions. The chatbot provides diagnoses and actionable steps. It also has a database of health information.

isabelhealthcare.com. It can be integrated with electronic medical record. Its paid clinical educator mode for physicians combines differential diagnosis generator with case histories [3]. It is useful for learning clinical reasoning and medical diagnosis.

Amazon.care. It is available to employees of Amazon and other companies. There are plans to substantially expand the system.

symptomate.com. It allows text chat or voice input through a digital voice assistant. Multilingual support is available. It is one of the few systems that can work on a phone as a voice-based application. More than four million persons have used it. 
Medicallyhome.com. It facilitates provision of acute care in extra-hospital settings. Mayo Clinic and Kaiser Permanente are utilizing it.

\section{Summary}

A rapidly increasing number of AI aided PDHAs are already being utilized by millions of persons. They are an integral part of telehealth. These systems are improving rapidly and are bound to play an everincreasing role in perioperative and other medical care.

Financial Disclosures: None

Conflicts of interest: None

\section{References}

1. Dr Google will see you now: Search giant wants to cash in on your medical queries.

2. Hospital Innovation models. (2020). CMS Announces Comprehensive Strategy to Enhance Hospital Capacity Amid COVID-19 Surge.

3. Semigran H, Linder J, Gidengil C, Mehrotra A. (2015). Evaluation of symptom checkers for self-diagnosis and triage: audit study. BMJ.

4. Riches N, Panagioti M, Alam R, Cheraghi-Sohi S, Campbell S, Esmail A, Bower P. (2016). The Effectiveness of Electronic Differential Diagnoses (DDX) Generators: A Systematic Review and Meta-Analysis. PLoS ONE. 11(3): e0148991. (c) $\oplus$

This work is licensed under Creative Commons Attribution 4.0 License

To Submit Your Article Click Here: Submit Manuscript

DOI: $10.31579 / 2768-2757 / 027$
Ready to submit your research? Choose Auctores and benefit from:

$>$ fast, convenient online submission

$>$ rigorous peer review by experienced research in your field

$>$ rapid publication on acceptance

$>$ authors retain copyrights

$>$ unique DOI for all articles

$>$ immediate, unrestricted online access

At Auctores, research is always in progress.

Learn more auctoresonline.org/journals/journal-of-clinical-surgery-andresearch 